\title{
Hollow waveguides as polarization converting elements: a theoretical study
}

S. F. Helfert

stefan.helfert@fernuni-hagen.de

\section{A. Edelmann}

\section{J. Jahns}

Chair of Micro- and Nanophotonics, FernUniversität in Hagen, Germany

Chair of Micro- and Nanophotonics, FernUniversität in Hagen, Germany

Chair of Micro- and Nanophotonics, FernUniversität in Hagen, Germany

Subwavelength apertures in a metallic film act as hollow waveguides. By using a non-quadratic cross-section, an anisotropic transmission behaviour results for the two polarization states. Thus, an array of metallic subwavelength apertures may be used as polarization converter, e.g., as a half-wave plate. By varying orientation and shape of the cross-sections locally, one can design polarization shifting elements for complex wave fields. Here, we present a theoretical consideration on the physical properties and compare with dielectric form birefringence. [DOI: http://dx.doi.org/10.2971/jeos.2015.15006]

Keywords: Birefringent materials, polarization conversion, hollow waveguides

\section{INTRODUCTION}

\subsection{Context}

A polarization converter transforms light of one well-defined polarization state into another. A well-known example is a quarter-wave plate that converts linearly polarised light to circular polarization and vice versa. A half-wave plate converts a linear $x$-polarised beam to a $y$-polarised beam. Polarization conversion can be achieved by different means, however, the principle always includes an anisotropy of light propagation. In classical optics, polarization converters use anisotropic crystals like crystalline quartz, for example [1], [2]. With the technical possibilities of micro- and nano-structuring, anisotropy can also be achieved artificially. Polarization conversion was demonstrated by using dielectric subwavelength gratings (see e.g. [3]-[5]). Further examples are L-shaped hole arrays [6] or chiral metamaterials [7]. Also liquid crystals have been used for the polarization conversion of optical waves as described e.g. in [8]-[11]. Here, we consider yet another approach, namely the use of light propagation in hollow metallic waveguides.

\subsection{Motivation}

Polarization conversion is widely necessary in optical systems applications. Some examples include heterodyne transmission systems, optical displays, interferometers, laser radar systems, etc., to mention just a few. A specific application that motivates our work here is the coupling of plasmons to metallic wires.

On metallic wires plasmon waves can be guided at Terahertz frequencies as shown experimentally [12] and theoret-
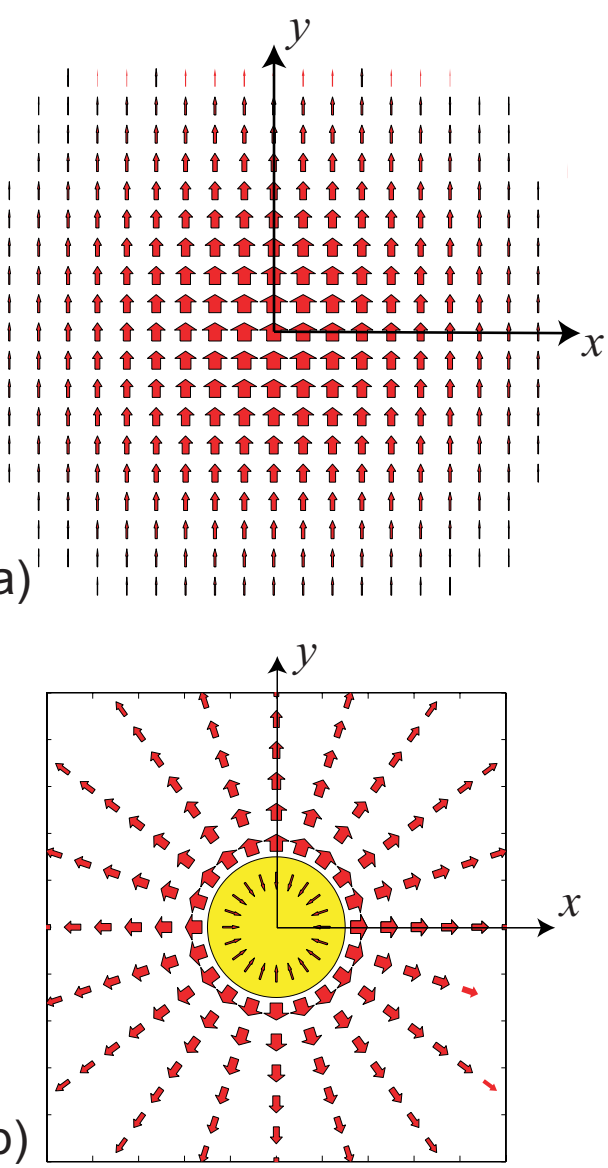

FIC. 1 Electric field distribution from the THz-source and of the Sommerfeld mode on an electric wire.

ically [13]. The excitation of such waves on metallic wires via end-fire coupling was studied experimentally in [14]. 
Figure 1(a) shows the electric field of a Gaussian beam coming from the THz-source, which shall be coupled to the metallic wire. The field of the guided eigenmode ("Sommerfeldmode") on the wire is presented in Figure 1(b). As can be seen, this Sommerfeld-mode is radially polarized and the two fields (THz-source, Sommerfeld-mode) have different symmetry characteristics with respect to the vertical axis. Hence, the Sommerfeld-mode cannot be excited with a symmetric injection. Therefore, either an asymmetry must be introduced or a polarization conversion has to be performed.

Polarization conversion is achieved in conventional optics by using components made of crystalline, hence anisotropic materials [15]. More recently, liquid crystal components became useful devices for polarization conversion [16] and even more recently, with the possibilities of nano-structuring, spacevariant polarization conversion has become feasible [3].

In this paper we want to exploit the birefringence in hollow waveguides to develop polarization converting elements. Compared with subwavelength gratings a greater difference of the effective index can be realized (for the same mean value). We expect an easier fabrication of the hollow waveguides in comparison to the L-shaped hole arrays [6].

As mentioned before, we are interested in improving the coupling of Terahertz waves to metallic wires. Although our motivation lies in THz-domain, it is also of interest to see if the approach with hollow waveguides might also be useful for optical frequencies. Besides, we can also perform optical experiments in our labs, to verify the theoretical findings experimentally. Therefore studies for $\mathrm{THz}$ - and optical-frequencies are done here.

The paper is organized as follows. We start with describing the principle of the polarization conversion via form birefringence. In the following main part of this paper, we deal with hollow waveguides. First, their main characteristics known from microwaves are summarized. After that, numerical studies at $\mathrm{THz}$ and optical frequencies are performed. We take a closer look at the eigenmodes and simulate the wave propagation resulting in a polarization conversion. The paper ends with a summary.

\section{POLARIZATION CONVERSION WITH BIREFRINGENT MEDIA}

In this section we repeat the principle of polarization conversion with birefringent media. For this purpose let us take a look at Figure 2. A plane wave is injected into a birefringent medium. As indicated, there are different values for the refractive index of the horizontally and the vertically polarized waves. Consequently, the electric field at the input is divided into these two components, a horizontal and a vertical one.

Now, the height $(h)$ of the birefringent medium is chosen in such a way that these two components experience a phase difference of $\pi$ at the output:

$$
k_{0} h\left(n_{\mathrm{h}}-n_{\mathrm{v}}\right)=\pi
$$

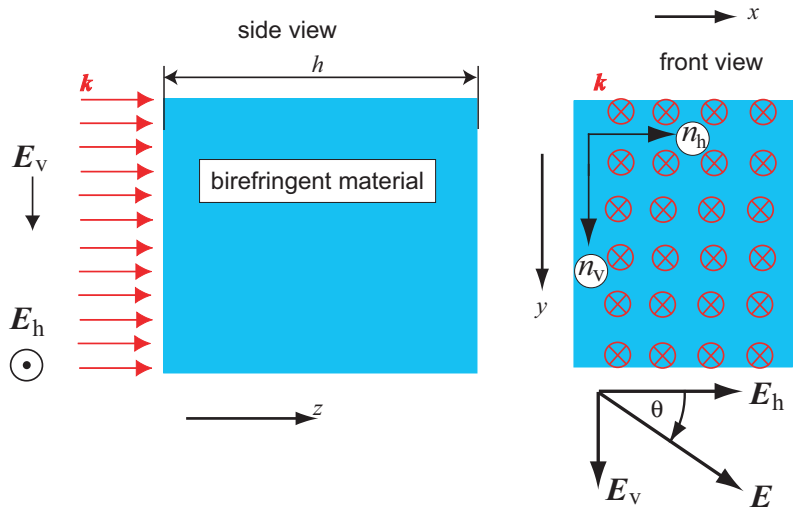

FIG. 2 Injection of a plane wave into a birefringent medium; the axes with different refractive indices $\left(n_{\mathrm{h}}\right.$ and $\left.n_{\mathrm{v}}\right)$ are indicated.
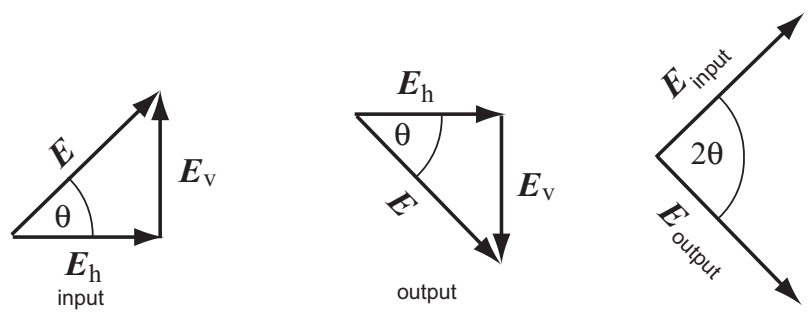

FIG. 3 Principle of the polarization conversion.

The principle of the polarization conversion is explained in Figure 3. On the left, the input field is divided into its horizontal and normal component. As described above, a phase difference of $\pi$ occurs at the output. So, when the horizontal component of the output field shows to the right, the vertical component points downwards, resulting in the sketched total field. On the right, a comparison of the input and the output field is shown. As can be seen, a wave that is polarized at the angle $\theta$ with respect to the horizontal axis is rotated by $2 \theta$.

To achieve a radial polarization that is required to excite the Sommerfeld-mode on the metallic wire, the birefringent material is oriented in different directions as shown on the top of Figure 4. A discrete number of sections (here $m_{\text {sect }}=4$ ) is assumed in this example. Now, a vertically polarized field is injected. Then, each of the sections causes an individual rotation, resulting in the output field shown at the bottom of Figure 4 . With increasing $m_{\text {sect }}$ the output field becomes "more radial polarized" and $m_{\text {sect }} \rightarrow \infty$ results in a continuos variation.

\section{HOLLOW WAVEGUIDES}

\subsection{Fundamentals}

In this paper we propose the use of arrays of hollow waveguides as polarization conversion elements. We will describe the main idea in this section. The cross-section of such an array is shown in Figure 5. The waves are guided inside the air region and the metallic walls act as mirrors. Single hollow waveguides are used to transmit signals over longer distances in the microwave area and their characteristics are explained in textbooks, (see e.g. [17]). Here, we start with describing their main features. 

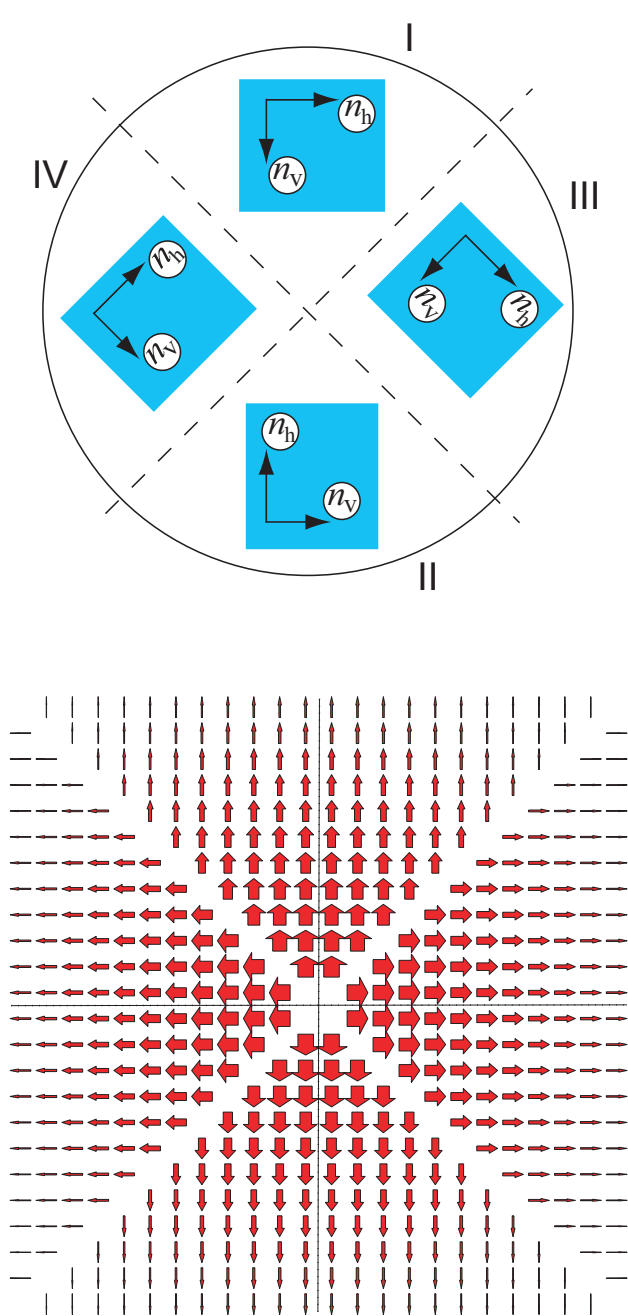

FIG. 4 Different orientation of the birefringent material in a discrete number of sections (here $m_{\text {sect }}=4$ ), and different polarization rotation in the different regions; the output field approaches a radial polarization.

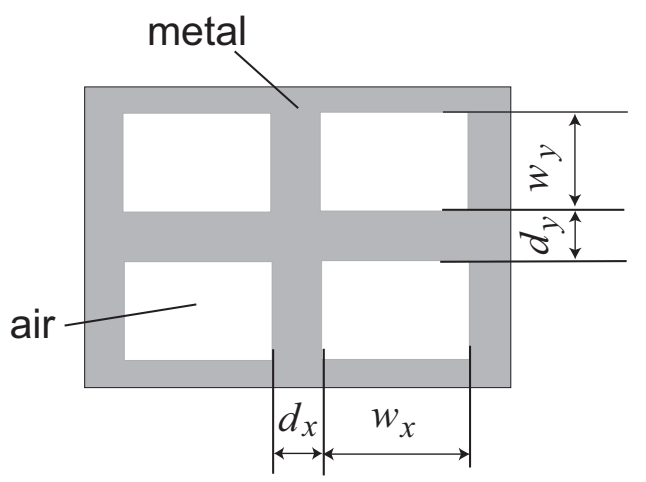

FIG. 5 Array of hollow waveguides with dimensions.

Hollow waveguides support various eigenmodes (labelled $\mathrm{TE}_{m n} \mathrm{TM}_{m n}$ or alternatively $H_{m n}$ resp. $\left.E_{m n}\right)$. The electric and magnetic fields of these eigenmodes can be determined from vector potentials. The subscripts $m, n$ correspond to the $x$ or $y$ dependency of the potentials ( $\psi_{h, e}$ see e.g. [17]), given as:

$$
\psi_{h, m n} \sim \cos \left(\frac{m \pi}{w_{x}} x\right) \cos \left(\frac{n \pi}{w_{y}} y\right)
$$

for the TE- (or $H$-) modes and

$$
\psi_{e, m n} \sim \sin \left(\frac{m \pi}{w_{x}} x\right) \sin \left(\frac{n \pi}{w_{y}} y\right)
$$

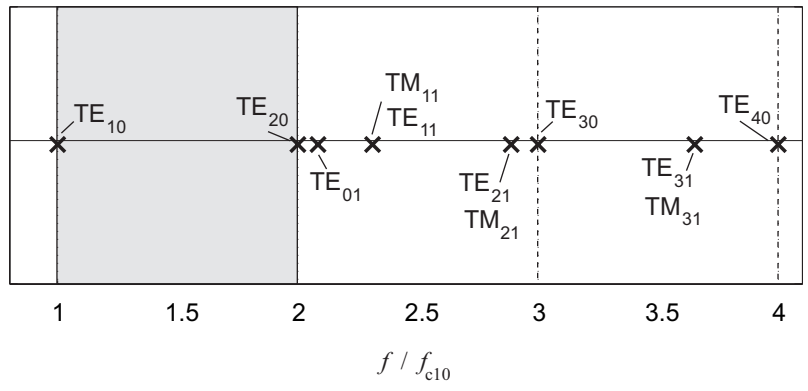

FIG. 6 Cut-off frequencies of the waveguide modes normalized with $f_{\mathrm{c} 10}$ for $w_{y}=0.48$ $w_{x}$.

for the TM- or (E-) modes, with

$$
0 \leq x \leq w_{x} \quad 0 \leq y \leq w_{y}
$$

Each of the eigenmodes has an own cut-off frequency. Only if the operating frequency is above this value, the mode is guided. The cut-off frequencies $f_{\mathrm{cm} n}$ are given as:

$$
f_{\mathrm{c} m n}=\frac{c_{0}}{2 \sqrt{\varepsilon_{\mathrm{r}}}} \sqrt{\frac{m^{2}}{w_{x}^{2}}+\frac{n^{2}}{w_{y}^{2}}}
$$

Here and in the following we assume $w_{x} \geq w_{y}$ and (as indicated in Figure 5) waveguides filled with air, i.e. $\varepsilon_{\mathrm{r}}=1$. Then, the fundamental mode is the $\mathrm{TE}_{10}$-mode with the cut-off frequency

$$
f_{\mathrm{c} 10}=\frac{c_{0}}{2 w_{x}}
$$

Hence, as can be easily shown, this mode is guided if the width $w_{x}$ is greater than half of the wavelength.

$$
w_{x}>\frac{\lambda_{0}}{2}
$$

In particular, we note, that the cut-off frequency does not depend on $w_{y}$. As we will see soon, the same is true for the effective index of this mode. This will allow us to design polarization converting components relatively easily.

The cut-off frequencies of the lowest order modes (normalized with $f_{\mathrm{c} 10}$ ) are shown in Figure 6 for the special case $w_{y}=0.48 w_{x}$ as example. (Note: due to the sinusoidal functions no $\mathrm{TM}_{m 0}$ (resp. $\mathrm{TM}_{0 n}$ ) exist.) Circuits in microwaves are usually designed for the fundamental mode. Hence, one usually chooses $w_{y}<w_{x} / 2$. Then, one can make sure that only the fundamental mode is guided in a maximum frequency range from $f_{\mathrm{c} 10}$ to $2 f_{\mathrm{c} 10}$. As can be seen from Eq. (2): for $f>2 f_{\mathrm{c} 10}=f_{\mathrm{c} 20}$ the $\mathrm{TE}_{20}$-mode always becomes guided.

To utilize hollow waveguides as polarization converters, it is important to note that all $\mathrm{TE}_{m 0}$-modes (particularly the fundamental mode) are vertically polarized. Since a polarization conversion shall be achieved, also a horizontally polarized guided mode is required here, which is the $\mathrm{TE}_{01}$-mode. Its characteristics are similar to those of the fundamental $\mathrm{TE}_{10^{-}}$ mode. E.g. to determine its cut-off frequency, we have to replace $w_{x}$ by $w_{y}$ in Eq. (3).

Next we look at the effective index. Generally, (i.e. for arbitrary modes of the hollow waveguides) it is determined as: 


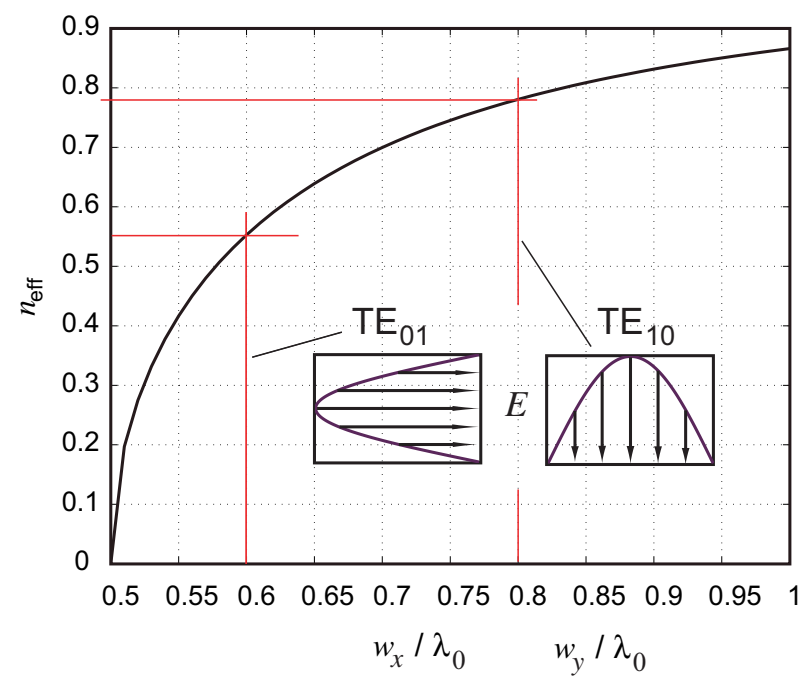

FIC. 7 Effective index of the $\mathrm{TE}_{01}\left(\mathrm{TE}_{10}\right)$ mode as function of $w_{x}\left(w_{y}\right)$; the inlay shows the electric field distribution of these modes.

$$
n_{\mathrm{eff}, m n}=\sqrt{\varepsilon_{\mathrm{r}}-\frac{1}{4}\left(\frac{m^{2} \lambda_{0}^{2}}{w_{x}^{2}}+\frac{n^{2} \lambda_{0}^{2}}{w_{y}^{2}}\right)}
$$

Then, the effective index of the fundamental mode is $\left(\varepsilon_{\mathrm{r}}=1\right)$ :

$$
n_{\mathrm{eff}, 10}=\sqrt{1-\frac{\lambda_{0}^{2}}{4 w_{x}^{2}}}
$$

By replacing $w_{x}$ with $w_{y}$ we obtain the effective index of the $\mathrm{TE}_{01}$-mode from this expression.

Let us summarize the features that we need to realize polarization conversion with hollow waveguides: By suitable choice of the dimensions, we can design a device that supports two guided modes, whose fields are perpendicular to each other. The effective index of these modes is determined by different parameters so that their values may be chosen independently. To visualize this, we take a look at Figure 7 . Here the effective index of the $\mathrm{TE}_{10^{-}}$(resp. $\left.\mathrm{TE}_{01^{-}}\right)$mode as function of $w_{x}\left(w_{y}\right)$ is shown [see Eq. (6)]. As example the values $w_{x}=0.8 \lambda_{0}$ and $w_{y}=0.6 \lambda_{0}$ were chosen. This results in the values 0.78 and 0.55 for the effective indices and a difference $\Delta n_{\text {eff }}=0.23$.

In practice, one would actually do the design in the opposite way: From a given $\Delta n_{\text {eff }}$ a suitable pair $w_{x}, w_{y}$ would be chosen. Since this choice is not unique, further considerations (e.g. from the fabrication process) would be taken into account.

\subsection{Eigenmodes of hollow waveguides for Terahertz and optical frequencies}

The features that were described in the last section are true for single waveguides at microwave frequencies, with a high conductivity of the metal. In this section we examine how the characteristics change due to the differing values for the material parameters at $\mathrm{THz}$ and optical frequencies. Also it is studied what happens when waveguide arrays are considered instead of single ones. As numerical tool the Method of Lines (see e.g. [18]) was used. Like before, the structure shown in

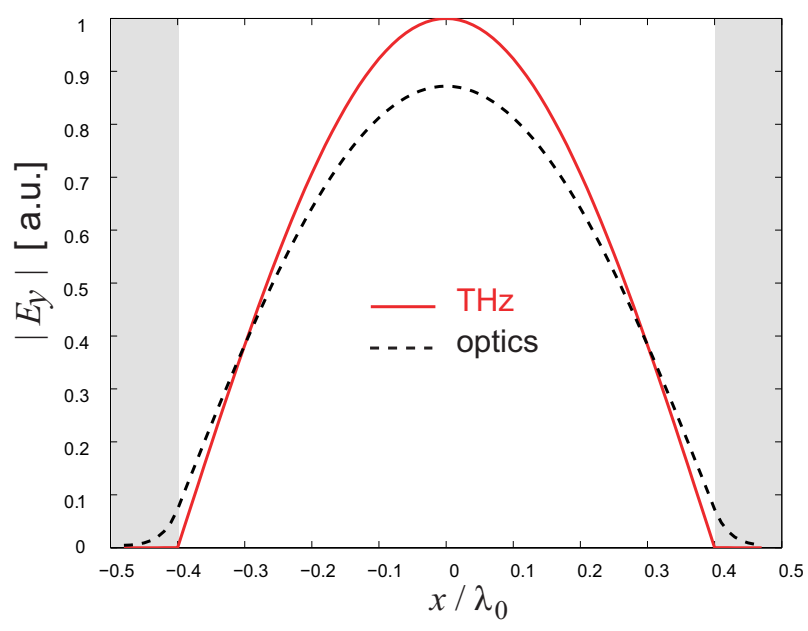

FIG. 8 Electric field distribution of the fundamental mode for optical and THz frequencies.

Figure 5 was examined. Unless stated otherwise, the values $w_{x}=0.8 \lambda_{0}, w_{y}=0.6 \lambda_{0}$ and $d_{x}=d_{y}=0.04 \lambda_{0}$ were taken. The metal was silver. Its relative permittivity $\left(\varepsilon_{\mathrm{rAg}}\right)$ was determined from a Drude model resulting in:

- $\mathrm{THz}\left(\lambda_{0}=0.48 \mathrm{~mm}, f=0.625 \mathrm{THz}\right)$

$$
\begin{array}{r}
\varepsilon_{\mathrm{rAg}}=-6.2 \times 10^{5}-2.25 \times 10^{6} j \\
\text { - Optics }\left(\lambda_{0}=1000 \mathrm{~nm}, f=3 \times 10^{14} \mathrm{~Hz}\right) \\
\varepsilon_{\mathrm{rAg}}=-47-1.89 j
\end{array}
$$

These special frequencies were chosen, because the $\mathrm{THz}$ coupling described in [14], was done for this frequency and due to experiments that will be performed in our lab for wavelengths around $1000 \mathrm{~nm}$.

Let us begin with a single waveguide. Figure 8 shows the electric field $\left(E_{y}\right)$ of the fundamental mode as function of $x$ normalised with the wavelength $\lambda_{0}$. In the $\mathrm{THz}$ case the field is zero inside the metal. This is the same result as in microwaves. The situation is different in optics, where the field extends quite far into the metal. From Figure 8 we see that it becomes zero in the metal at $\approx 0.1 \lambda_{0}$. This behaviour has also consequences for the effective index as shown in Figures 9 and 10. First of all, we notice that the effective index in optics is higher than in the THz-case. It is not shown here, but we would like to mention that also losses (determined from the imaginary part of $n_{\text {eff }}$ ) are higher for optical frequencies.

Now, the effective index as function of $w_{x}$ with $w_{y}$ as parameter is presented in Figure 9. All curves are on top of each other in the THz-regime, while a slight decrease with increasing $w_{y}$ occurs in optics. This can be seen more clearly in Figure 10 where the effective index is shown as function of $w_{y}$ (with $w_{x}$ as parameter). The $\mathrm{THz}$-curves run horizontally, showing that $n_{\text {eff }}$ is independent of $w_{y}$. In optics, however, a decrease of the curves can be observed. Hence, the effective index here depends on both parameters $w_{x}$ and $w_{y}$.

After looking at a single waveguide, an arrays with $2 \times 2$ waveguides was studied. Electric field distributions of the 


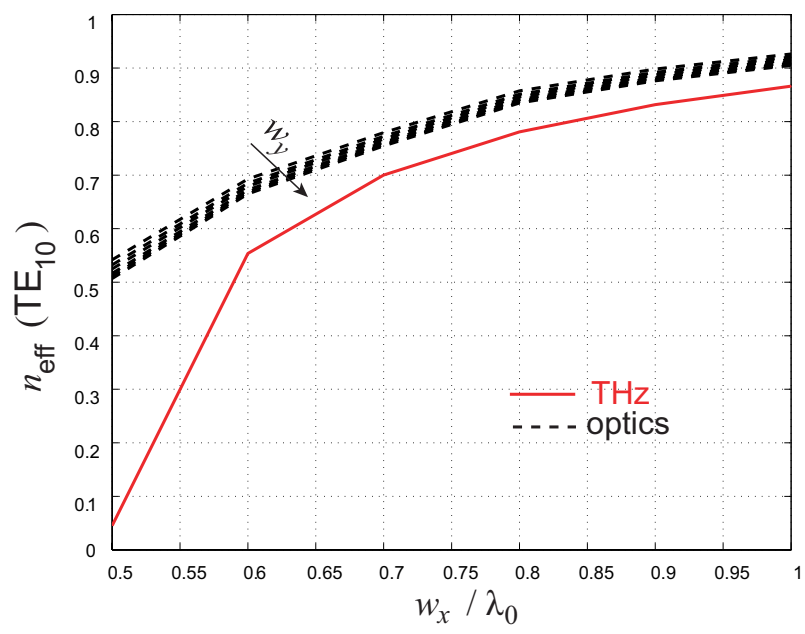

FIG. 9 Effective index of the fundamental mode as function of $w_{x}$.

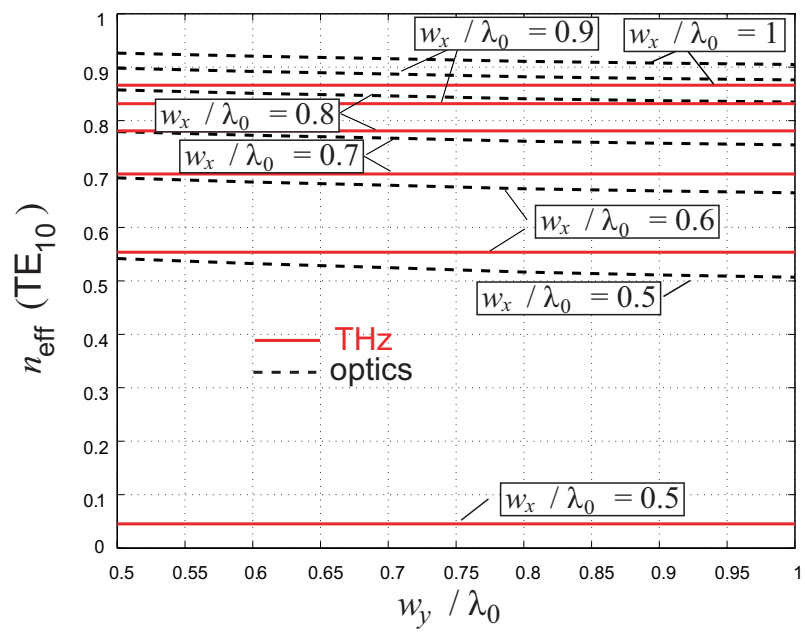

FIG. 10 Effective index of the fundamental mode as function of $w_{y}$.

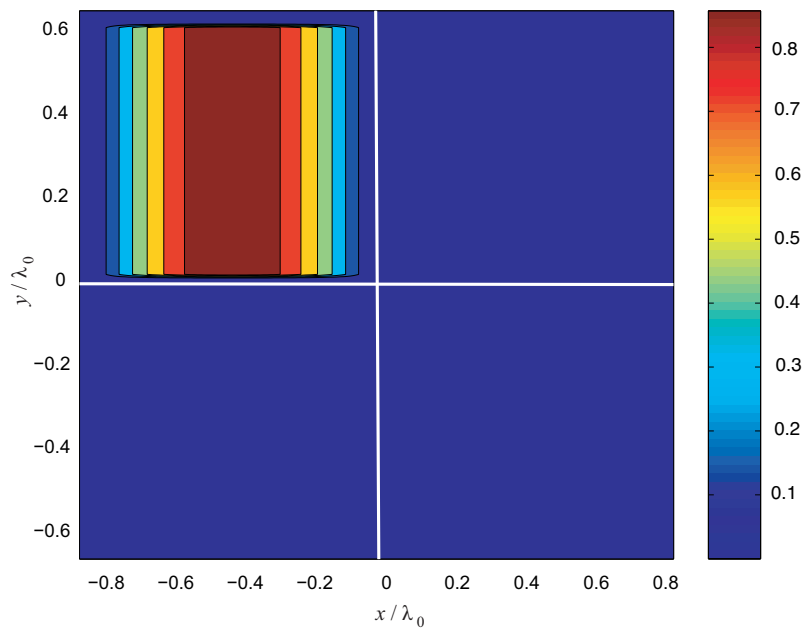

FIC. 11 Electric field distribution $\left(E_{y}\right)$ in a waveguide array for THz-waves.

eigenmodes for the arrays are shown in Figures 11 and 12. In the THz-case the field of all eigenmodes covers only one of the single waveguides. As example the field in the upper left waveguide is shown in Figure 11. As in microwaves, we see a cosine wave form in $x$-direction and constant values in $y$-direction. The eigenmodes covering the other waveguides have the same shape and the values for the effective index are identical.

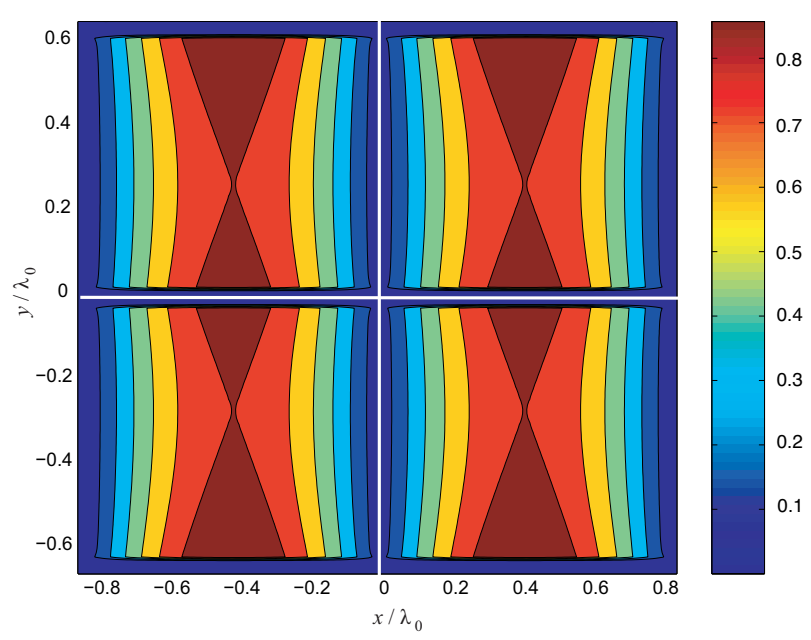

FIG. 12 Electric field distribution $\left(E_{y}\right)$ in a waveguide array (optics).

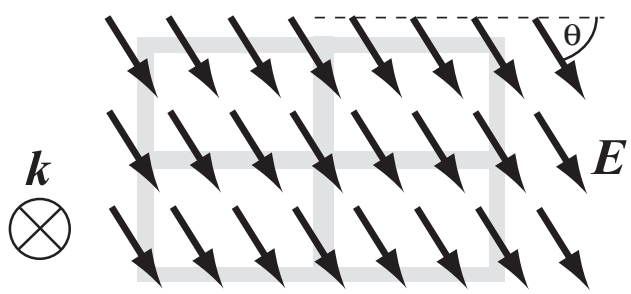

FIG. 13 Injection of a plane wave into an array of hollow waveguides.

The situation is different in optics as shown in Figure 12. As can be seen, the fields in all waveguides are coupled and they depend on the vertical coordinate $y$ in contrast to the THzcase. As example the symmetric (with respect to $x$ and $y$ ) eigenmode is shown. This is the mode that will be excited by a plane wave. The other supermodes have a similar shape but different symmetries. All supermodes have individual effective indices which also differ from those of the single guides. These results show that the findings for a single waveguide can be transferred immediately to waveguide arrays in the $\mathrm{THz}$ regime. In contrast, more complicated 3D-computations of the whole array have to be done in optics.

\subsection{Polarization conversion with hollow waveguides}

After studying the eigenmodes, we simulated the polarization conversion effect. For this purpose a tilted plane wave was injected into a waveguide array as indicated in Figure 13. The height of the array was determined from Eq. (1), where we used the effective indices of the relevant supermodes in optics. This results in:

$$
h_{\mathrm{THz}}=2.2 \lambda_{0} \quad h_{\mathrm{optic}}=3 \lambda_{0}
$$

for the $\mathrm{THz}$ and optical frequency.

Figure 14 shows the power at the output of the waveguide array as function of the angle $\theta$ (see Figure 13) for both- $\mathrm{THz}$ and optical fields. As expected, the whole power is in the horizontally (vertically) polarized modes for $\theta=0^{\circ}\left(90^{\circ}\right)$. For $\theta=45^{\circ}$ the power in the two polarizations is identical. The total power varies only little with $\theta$. So, in principle an an ar- 


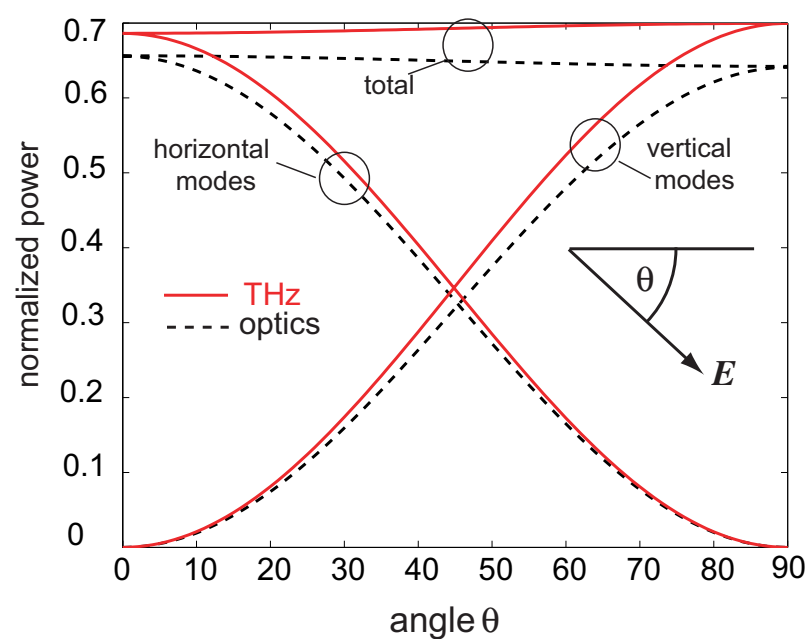

FIG. 14 Normalied power at the output of the hollow waveguide arrays.

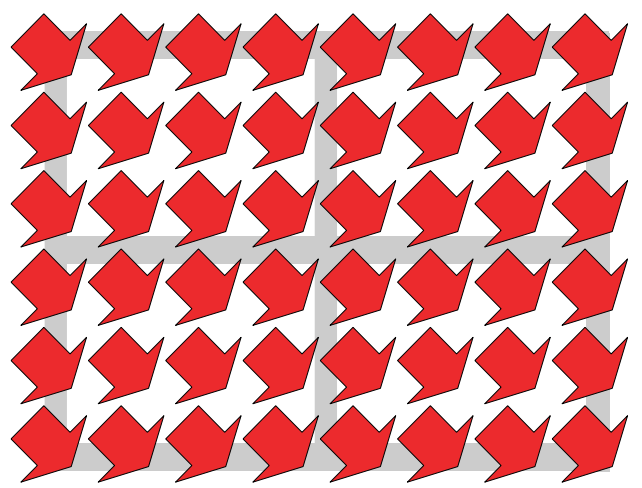

FIG. 15 Electric field distribution at the input of the hollow waveguide arrays.

bitrary number of sections (see Figure 4) can be introduced to create a radially polarized field.

The simulated field distributions for the input angle $\theta=45^{\circ}$ are shown in Figures 15-17. Figure 15 presents the input field of a plane wave and the field at the output of the waveguide is sketched in Figures 16 and 17. For both frequencies the rotation of the fields is observed. Unlike the input field, the output fields are not constant across the cross-section, because of the shape of the eigenmodes discussed earlier. A $90^{\circ}$ polarization conversion occurs in the middle of the waveguides whereas in the vicinity of the metal the fields are orientated perpendicular to the boundaries. The THz- and optics- graphs look very similar with small differences close to the metal boundaries. The numerical results show that the proposed polarization conversion with hollow waveguides succeeds theoretically. The next step is the fabrication of the devices and the experimental confirmation.

\section{CONCLUSIONS}

In this paper we studied arrays of hollow waveguides in the Terahertz and optical regime and their possible use as polar-

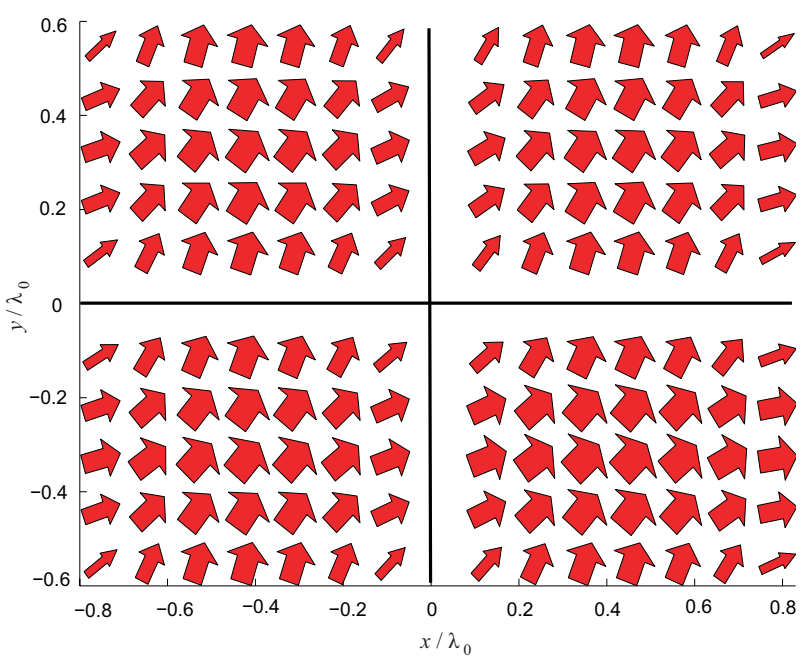

FIG. 16 Electric field distribution at the output of the hollow waveguide, (THz).

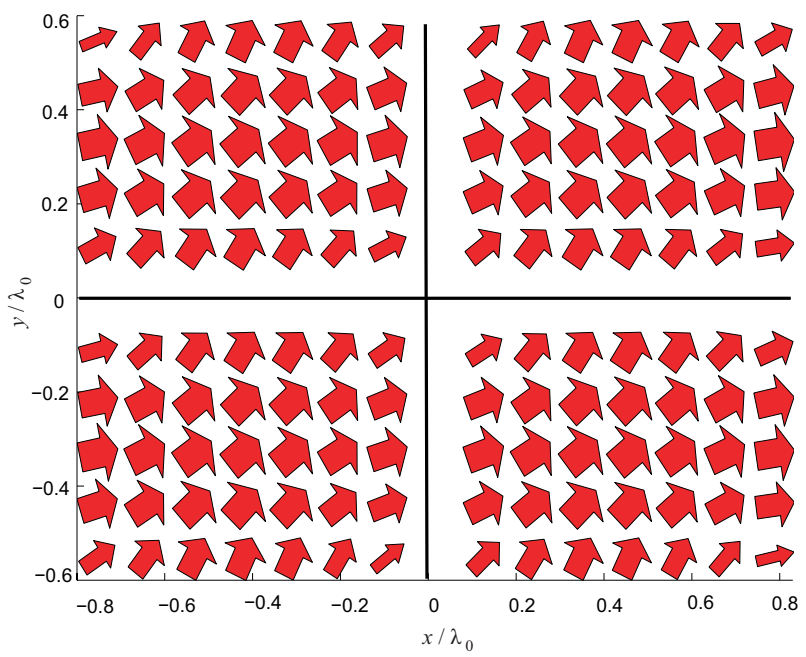

FIC. 17 Electric field distribution at the output of the hollow waveguide (optics).

ization converting elements. For the THz-regime, it was found that these structure behave very much like known from microwave theory. For the polarization conversion, waves with perpendicular polarizations must propagate with different effective indices. In the THz-regime such a difference can be tailored very easily, because $n_{\text {eff }}$ of the excited eigenmodes (the $\mathrm{TE}_{10}$ resp. $\mathrm{TE}_{01}$ ) depends on different parameters i.e. the width $w_{x}$ resp. $w_{y}$.

As seen before, in optics the field confinement is weaker. As consequence, the fields in the various single waveguides are coupled. Also both geometric dimensions $\left(w_{x}\right.$ and $\left.w_{y}\right)$ determine the effective index of the eigenmodes. An accurate description requires the simulation of the whole waveguide array with full 3D-algorithms.

For both frequencies ( $\mathrm{THz}$ - optics) a polarization conversion was observed. Compared to e.g. subwavelength gratings a larger contrast of the effective index for the two perpendicular polarizations can be achieved. Therefore, the height of the structures is much smaller. Nevertheless, the structures are technologically very demanding, because the ratio between the height and the thickness of the metal can be on the order of 10 to 50 . 


\section{References}

[1] P. B. Phua, W. J. Lai, Y. L. Lim, K. S. Tiaw, B. C. Lim, H. H. Teo, and M. H. Hong, "Mimicking optical activity for generating radially polarized light," Opt. Lett. 32 376-378 (2007).

[2] A. K. Kaveev, G. I. Kropotov, E. V. Tsygankova, I. A. Tzibizov, S. D. Ganichev, S. N. Danilov, P. Olbrich, C. Zoth, E. G. Kaveeva, A. I. Zhdanov, A. A. Ivanov, R. Z. Deyanov, and B. Redlich, "Terahertz polarization conversion with quartz waveplate sets," Appl. Optics 52 B60-B69 (2013).

[3] E. Hasman, V. Kleiner, G. Biener, and A. Niv, "Space-variant polarization-state manipulation with computer-generated subwavelength gratings," Proc. SPIE 4984, 171-185 (2003).

[4] G. M. Lerman and U. Levy, "Generation of a radially polarized light beam using space-variant subwavelength gratings at $1064 \mathrm{~nm}$," Opt. Lett. 33 2782-2784 (2008).

[5] S. Rothau, I. Harder, 0. Lohse, and N. Lindlein, "Künstliche nanostrukturierte Polarisationselemente," in DGAO-Proc., 114 B6 (Deutsche Gesellschaft für angewandte Optik e.V., Braunschweig, 2013).

[6] L. Wang, S. Jiang, H. Hu, H. Song, W. Zeng, and Q. Gan, "Artificial birefringent metallic planar structures for terahertz wave polarization manipulation," Opt. Lett. 39 311-314 (2014).

[7] Z. Ozer, F. Dincer, M. Karaaslan, and 0. Akgol, "Asymmetric transmission of linearly polarized light through dynamic chiral metamaterials in a frequency regime of gigahertz-terahertz," Opt. Eng. 53 075109 (2014).

[8] M. Stalder and M. Schadt, "Linearly polarized light with axial symmetry generated by liquid-crystal polarization converters," 0 pt. Lett. 21 1948-1950 (1996).
[9] S. Quabis, R. Dorn, and G. Leuchs, "Generation of a radially polarized doughnut mode of high quality," Appl. Phys. B - Lasers 0.81 597-600 (2005).

[10] H. Ren, Y.-H. Lin, and S.-T. Wua, "Linear to axial or radial polarization conversion using a liquid crystal gel," Appl. Phys. Lett. 89 051114 (2006).

[11] G. Machavariani, Y. Lumer, I. Moshe, A. Meir, and S. Jack, "Efficient extracavity generation of radially and azimuthally polarized beams," Opt. Lett. 32 1468-1470 (2007).

[12] K. Wang and D. M. Mittleman, "Metal wires for terahertz wave guiding," Nature 432 379-379 (2004).

[13] Q. Cao and J. Jahns, "Azimuthally polarized surface plasmons as effective terahertz waveguides," Opt. Express 13 511-518 (2005).

[14] A. Edelmann, L. Möller, and J. Jahns, "Coupling of terahertz radiation to metallic wire using end-fire technique," Electron. Lett. 49 884-886 (2013).

[15] E. Hecht, Optics, 4th ed. (Addison-Wesley, San Francisco, 2001).

[16] S. Masuda, T. Nose, and S. Sato, "Optical properties of a polarization converting device using a nematic liquid crystal cell," opt. Rev. 2 211-216 (1995).

[17] R. E. Collin, Field Theory of Guided Waves, 2nd ed. (IEEE press, New York, 1991).

[18] R. Pregla, Analysis of Electromagnetic Fields and Waves - The Method of Lines (Wiley it Sons, Chichester, UK, 2008). 